\title{
Emission Line Ratios for the Circumgalactic Medium and the "Bimodal" Nature of Galaxies
}

\author{
Huanian Zhang (张华年) ${ }^{1}$ (D), Dennis Zaritsky ${ }^{1}$ (D), Jessica Werk ${ }^{2}$ (D), and Peter Behroozi ${ }^{1}$ (D) \\ ${ }^{1}$ Steward Observatory, University of Arizona, Tucson, AZ 85719, USA; fantasyzhn@email.arizona.edu \\ ${ }^{2}$ Department of Astronomy, University of Washington, Seattle, WA 98195 , USA \\ Received 2018 August 16; revised 2018 September 18; accepted 2018 September 20; published 2018 October 4
}

\begin{abstract}
We find significantly different diagnostic emission line ratios for the circumgalactic gas associated with galaxies of stellar masses above and below $10^{10.4} M_{\odot}$ using Sloan Digital Sky Survey spectroscopy. Specifically, in a sample of 17,393 galaxies, intersected by 18,535 lines of sight at projected radii between 10 and $50 \mathrm{kpc}$, we stack measured fluxes for nebular strong emission lines, [O III] $\lambda 5007, \mathrm{H} \alpha$, and $[\mathrm{N} \mathrm{II}] \lambda 6583$, and find that the gas surrounding the lower-mass galaxies exhibits similar line ratios to those of gas ionized by star formation and that surrounding the higher-mass galaxies similar to those of gas ionized by active galactic nucleus or shocks. This finding highlights yet another characteristic of galaxies that is distinctly different above and below this stellar mass threshold, but one that is more closely connected to the gas accretion behavior hypothesized to be responsible for this dichotomy.
\end{abstract}

Key words: galaxies: halos - galaxies: kinematics and dynamics - galaxies: structure - intergalactic medium

\section{Introduction}

The manner in which gas accretes onto galaxies, and therefore the initial state of the gas observed in the circumgalactic medium (CGM), is hypothesized to be distinctly different in high- and low-mass galaxies (Birnboim \& Dekel 2003; Kereš et al. 2005). The physical distinction centers on whether the gas is shocked as it is accreted onto the dark matter galaxy halo, as originally envisioned by White \& Rees (1978), or directly accreted onto the central galaxy. The two modes are broadly referred to as "hot" and "cold" modes of accretion, respectively. The different behavior is expected to lead to distinguishing observable galaxy characteristics (Kereš et al. 2005; Dekel \& Birnboim 2006). Many of the key broad differences among galaxies, such as color, morphology, and current star formation rate (SFR), have long been known to track the mass of the galaxy (Roberts \& Haynes 1994) and that connection has been unambiguously demonstrated using data from the Sloan Digital Sky Survey (SDSS; Blanton et al. 2003; Kauffmann et al. 2003b). In particular, Kauffmann et al. (2003b) found that galaxies separate into "two distinct families" and that the break in galaxy properties happens relatively sharply at a stellar mass, $M_{*}$, of $\sim 3 \times 10^{10} M_{\odot}$, which corresponds closely to the transition scale for accretion behavior identified in numerical simulations, $M_{*}=10^{10.4} M_{\odot}$ (Kereš et al. 2005).

Since these studies, there has been an explosion in studies of the CGM (see Tumlinson et al. 2017, for a review with an extensive list of references). The emerging picture is far more complicated, including gas recycling, the contribution of gas from galaxy mergers, and even the accretion of gas processed previously in other galaxies (Ford et al. 2014; Anglés-Alcázar et al. 2017). There are even proposals that the current state of the gas may reflect a previous state of the central galaxy (for example, previous active galactic nucleus (AGN) activity; Oppenheimer et al. 2018). While no simple picture will capture all of this richness, there continue to be predictions that the properties of the CGM should depend on galaxy mass.
To bridge the gap between the measured central properties of galaxies and the theoretical modeling of gas accretion, we investigate whether the observed circumgalactic gas also differs significantly for low- and high-mass galaxies. Diagnostic emission line ratios, like those used in the Baldwin, Phillips \& Terlevich (BPT) diagram (Baldwin et al. 1981), provide guidance on the ionizing source. Those authors identified regions in line ratio diagrams indicative of excitation by normal star formation (H II regions), AGNs (which are power-law spectrum sources), shock heating, and hot stellar remnants (planetary nebulae). Subsequent studies that focused on the use of such line ratios to interpret galaxy spectra focused on distinguishing between the two expected dominant sources of excitation, star formation, and AGN (e.g., Veilleux \& Osterbrock 1987; Kewley et al. 2001; Kauffmann et al. 2003a).

Our observational challenge is to detect the line emission from the CGM necessary to construct the diagnostic ratios. Stacking thousands to millions of spectra obtained from galaxy redshift surveys has proven to provide an avenue for studying difficult-to-reach spectral features in a variety of contexts (e.g., Steidel et al. 2010; Bordoloi et al. 2011; Ménard et al. 2011; Zhu \& Ménard 2013a, 2013b; Werk et al. 2014, 2016; Croft et al. 2016, 2018; Prochaska et al. 2017; Joshi et al. 2018; Lan \& Mo 2018). We recently applied the stacking technique to SDSS spectra to uncover emission lines originating in the CGM of low-redshift galaxies (Zhang et al. 2016, 2018, hereafter, Papers I and II). These two studies present results on the radial distribution of $\mathrm{H} \alpha+[\mathrm{N}$ II] emission from the halos of normal galaxies and their neighbors to projected radii beyond $100 \mathrm{kpc}$. We continue our exploration of this gas using spectral stacking by now measuring additional recombination lines in an effort to constrain the physical state of the gas in the warm ionized halos of low- and high-mass galaxies using standard diagnostic line ratios. To evaluate distances, we adopt standard cosmological parameters $\Omega_{m}=0.3, \Omega_{\Lambda}=0.7, \Omega_{k}=0$ and the dimensionless Hubble constant $h=0.7$. 
Table 1

The Emission Fluxes for $\mathrm{H} \beta$, [O III], $\mathrm{H} \alpha$ and [N II] at Different Projected Radius

\begin{tabular}{|c|c|c|c|c|}
\hline $\begin{array}{l}r_{p}{ }^{\mathrm{a}} \\
{[\mathrm{kpc}]}\end{array}$ & \multicolumn{4}{|c|}{$\left[10^{-17} \mathrm{erg} \mathrm{cm}^{-2} \mathrm{~s}^{-1} \AA^{-1}\right]$} \\
\hline & \multicolumn{4}{|c|}{ Full Sample $\left(10^{9}<M_{*}<10^{11.75}\right)$} \\
\hline \multirow[t]{2}{*}{35} & $0.0017 \pm 0.0013$ & $0.0046 \pm 0.0013$ & $0.0054 \pm 0.0012$ & $0.0037 \pm 0.0012$ \\
\hline & \multicolumn{4}{|c|}{$10^{9}<M_{*} \leqslant 10^{10.4} M_{\odot}$} \\
\hline 35 & $0.0015 \pm 0.0017$ & $0.0043 \pm 0.0016$ & $0.0075 \pm 0.0016$ & $<0.0014 \pm 0.0015$ \\
\hline 17 & $\cdots$ & $0.012 \pm 0.004$ & $0.022 \pm 0.004$ & $0.0042 \pm 0.0036$ \\
\hline \multirow[t]{2}{*}{40} & $\cdots$ & $0.0027 \pm 0.0018$ & $0.0045 \pm 0.0017$ & $<0.00054 \pm 0.0017$ \\
\hline & \multicolumn{4}{|c|}{$10^{10.4}<M_{*}<10^{11.75} M_{\odot}$} \\
\hline 35 & $0.0019 \pm 0.0020$ & $0.0059 \pm 0.0019$ & $0.0028 \pm 0.0019$ & $0.0089 \pm 0.0018$ \\
\hline 17 & $\cdots$ & $0.024 \pm 0.005$ & $0.018 \pm 0.006$ & $0.021 \pm 0.005$ \\
\hline 40 & $\cdots$ & $0.0026 \pm 0.0021$ & $<0.0019 \pm 0.0019$ & $0.0067 \pm 0.0019$ \\
\hline
\end{tabular}

Note.

${ }^{\text {a }}$ Radii refer to the mean $r_{p}$ of lines of sight included. A value of $35 \mathrm{kpc}$ corresponds to a bin that includes all lines of sight with $r_{p}$ between 10 and $50 \mathrm{kpc}$. Values of 17 and 40 correspond to inner and outer $r_{p}$ bins within that range, respectively.

\section{Data Analysis}

We follow the approach developed in Papers I and II, but focus on the CGM of host galaxies by considering only sightlines with projected separations $\leqslant 50 \mathrm{kpc}$ (see Paper II for a discussion of how neighboring halos begin to dominate the integrated emission profile at larger projected radii). We obtain galaxy spectra from the SDSS Data Releases (DR12; Alam et al. 2015) and classify galaxies that meet our criteria in redshift $(0.02<z<0.2)$, luminosity $\left(10^{9.5} \leqslant L / L_{\odot}<10^{11}\right)$, and size $\left(2 \leqslant R_{50} / \mathrm{kpc}<10\right)$ as candidate primary galaxies. We then compile SDSS spectroscopic lines of sight to other galaxies that are projected within $50 \mathrm{kpc}$ of any candidate primary galaxy to probe the CGM of the primary galaxy. To avoid contamination from satellites and other nearby galaxies, we require a redshift difference from the primary $>0.05$. For each such spectrum, we fit and subtract a tenth order polynomial to a $200 \AA$ wide section at the rest wavelength, in the primary galaxy frame, for each of the emission lines of interest to remove the continuum. The emissions lines that we study are $\mathrm{H} \beta,[\mathrm{O} \mathrm{III}] \lambda 5007, \mathrm{H} \alpha$, and [N II] $\lambda 6583$. For completeness, we also measure and detect [O II] $\lambda \lambda 3726,3729$ at $>3 \sigma$ significance, but we do not discuss it further because it is not needed for the diagnostic line ratios that we discuss below.

We measure the emission flux within a prescribed velocity window relative to the primary galaxy from each individual spectrum and combine the measurements. For simplicity, we set the width of that velocity window, $\pm 180 \mathrm{~km} \mathrm{~s}^{-1}$, to approximate the range of kinematics expected in the halos of massive galaxies. This choice will include most of the absorption line systems in halos (Werk et al. 2016). However, we also experiment with a variable width related to an estimate of the virial velocity and describe those results, which are qualitatively similar to those obtained with the fixed window below. Details of how we process the data, including the rejection of outliers, are detailed in Papers I and II. In those papers, we adopted a broader velocity window than we do here to include as much signal as possible because we pursue detections to large projected radii and, therefore, cannot separately measure $\mathrm{H} \alpha$ and [N II]. Finally, we extract $M_{*}$ (Kauffmann et al. 2003a, 2003b; Gallazzi et al. 2005) and SFRs (Brinchmann et al. 2004) from the MPA-JHU catalog.

\section{Results}

We present measurements of the mean line fluxes of the different emission lines and the associated uncertainties, calculated using the dispersion among individual measurements, for lines of sight with projected radius, $r_{p}$, between 10 and $50 \mathrm{kpc}$ in Table 1 . The mean $r_{p}$ for the lines of sight within this $r_{p}$ range is $35 \mathrm{kpc}$, well within the halos of most galaxies. Estimates of the virial radius for these galaxies, obtained as explained below, range from $\sim 70$ to $500 \mathrm{kpc}$, although most are not as extreme and much closer to the mean, $270 \mathrm{kpc}$.

We detect statistically significant, $>3 \sigma$, flux for all of our targeted lines in the full sample except for $\mathrm{H} \beta$ (top line of Table 1). ${ }^{3}$

\subsection{BPT Diagram}

The BPT diagnostic diagram is constructed using two line ratios, $[\mathrm{O} \mathrm{III}] / \mathrm{H} \beta$ and $[\mathrm{N} \mathrm{II}] / \mathrm{H} \alpha$, to define a parameter space in which one can distinguish between softer ionization sources, star formation principally, and harder sources, power law (AGN), or shocks. The use of $\mathrm{H} \beta$ in one of the ratios and $\mathrm{H} \alpha$ in the other is driven by the desire to avoid different extinction corrections between the numerator and denominator in those ratios. This condition is satisfied because $\mathrm{H} \beta$ is close in wavelength to [O III] $\lambda 5007$ and $\mathrm{H} \alpha$ is close in wavelength to [N II] $\lambda 6583$.

The $\mathrm{H} \beta$ null detection is therefore unfortunate. The theoretical expectation for $\mathrm{H} \beta / \mathrm{H} \alpha$ has some variation that depends on physical conditions. Calculations can be done both for Case A or Case B (e.g., Baker \& Menzel 1938; Hummer \& Storey 1987; Osterbrock \& Ferland 2006), which describe whether one assumes that the gas is or is not optically thin, respectively, in the Lyman lines. Case B is typically assumed for gas in galaxies, although that gas is generally much denser than the gas in halos. Such calculations predict ratios of $\sim 0.35$. The $\mathrm{H} \beta / \mathrm{H} \alpha$ flux ratio that we measure is $0.31 \pm 0.25$, which is entirely consistent with these expectations, but is sufficiently uncertain that it provides no discriminatory power.

\footnotetext{
3 The conversion factor to units between the values that we present, $10^{-17} \mathrm{erg} \mathrm{cm}^{-2} \mathrm{~s}^{-1} \AA^{-1}$ and those used commonly in the literature to describe diffuse line emission, erg $\mathrm{cm}^{-2} \mathrm{~s}^{-1} \operatorname{arcsec}^{-2}$, is 1.7 .
} 


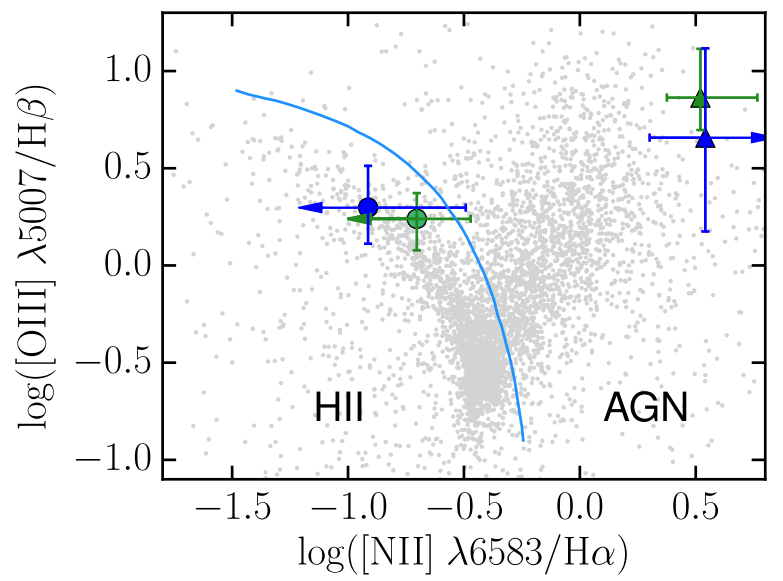

Figure 1. BPT diagram for circumgalactic gas within $10<r_{p} / \mathrm{kpc}<50$ (mean projected radius $=35 \mathrm{kpc}$; green symbols) and within a larger radius bin $22<r_{p} / \mathrm{kpc}<50$; blue symbols). The circle and triangle represent the measurements for galaxies with stellar mass below and above $10^{10.4} M_{\odot}$, respectively. The blue curve is the demarcation between ratios indicating ionization by star formation and AGN/shocks (Kauffmann et al. 2003a), with labels indicating which region corresponds to each of the two mechanisms. The light gray points represent the line ratios for the integrated central parts of individual galaxies as measured by SDSS. The $\mathrm{H} \beta$ flux is estimated using the $\mathrm{H} \alpha$ flux as described in the text.

Because we do not have a statistically robust determination of $\mathrm{H} \beta$, we cannot present the standard BPT diagram. Instead, we estimate $\mathrm{H} \beta$ using $\mathrm{H} \alpha$ and an adopted $\mathrm{H} \beta / \mathrm{H} \alpha$ line ratio. Differential extinction between $\mathrm{H} \alpha$ and $\mathrm{H} \beta$ is not an issue here as extinction in galaxy halos is exceedingly low (Zaritsky 1994; Ménard et al. 2010). To be specific, Ménard et al. (2010) found that $A_{V}$ is less than 0.03 beyond projected radii of $15 \mathrm{kpc}$ in their generic galaxy and is $\sim 0.01$ at the mean $r_{p}$ of our sample, $35 \mathrm{kpc}$. The use of a correction factor between $\mathrm{H} \alpha$ and $\mathrm{H} \beta$ does introduce a source of uncertainty, but that can be mitigated by considering limiting cases. We choose to adopt $\mathrm{H} \beta / \mathrm{H} \alpha=0.3$, a round number consistent with our measurement for the entire sample and theoretical expectations. In almost all scenarios, one expects the ratio to be larger than this value and, hence, the calculated ratios may slide in one direction (downward) along the $y$-axis in our BPT diagrams.

We derive the errorbars plotted in Figure 1 using a bootstrapping method. Specifically, we randomly select half of the individual measurements in each of the two mass subsamples, calculate the mean fluxes for $\mathrm{H} \alpha, \mathrm{N}[\mathrm{II}]$ and $\mathrm{O}[\mathrm{III}]$, evaluate the ratios, treat negative ratio values as described above, and repeat the process 1000 times. We define the 16.5 and 83.5 percentiles of the resulting ratio distributions as the ends of the plotted errorbars. The ratio values we present are the medians of the distributions. In Figure 2, we present the stacked composite spectra in the wavelength region of $\mathrm{H} \alpha$ and $\mathrm{N}[\mathrm{II}]$ for lines of sight with $10<$ $r_{p}<50 \mathrm{kpc}$ for both low- and high-mass subsamples. The $\mathrm{H} \alpha$ signal is high in low-mass subsample while the $\mathrm{N}$ [II] signal is high in the high-mass subsample, as expected from Figure 1.

To estimate the statistical significance of our result, we perform a bootstrap analysis by randomly splitting the sample in two, matching in size to our low- and high-mass subsamples. Among 1000 trials, we find no cases where the mean subsample line ratios match the observed values or where the lower-mass sample is even farther toward the lower left in the diagram, and the high-mass sample farther toward the upper right, which suggests that the observational result is significant at $\geqslant 99.9 \%$ confidence. For a more conservative determination, however, we measure how often we find either of the two subsamples lying on a different side of the $\mathrm{HII} / \mathrm{AGN}$ demarcation line than the other subsample. Here, we find an incidence of $4 \%$, suggesting $>95 \%$ confidence in our result. These two estimates are likely to bracket the actual significance level.

One concern in interpreting our result is that we have mixed measurements from different radial regimes, the innermost of which may be contaminated by emission from the central galaxy. To address this concern, we divide the data into two equal $\Delta \log r_{p}$ bins centered on $17\left(10<r_{p} / \mathrm{kpc}<22.4\right)$ and $40 \mathrm{kpc}\left(22.4 \leqslant r_{p} / \mathrm{kpc}<50\right)$. We present the mean flux value for each line, the uncertainty of the mean, and the average $r_{p}$ within each bin in Table 1. Because of the smaller sample size once we split the sample, uncertainties are larger, and in two cases we measure a non-positive flux (less than $1 \sigma$ at or below 0). To enable us to present those in the log space of the BPT diagram, at least as upper limits, we added $1 \sigma$ to the measured values. The two cases where that happened are listed as upper limits in Table 1. The diagnostic line ratios are consistent from the inner to outer bin in both the low- and highstellar mass samples. We show the larger radius bin results in Figure 1 for comparison to the results using the full sample. The use of the adjusted values from the limits does not affect this conclusion because in both cases using even smaller flux values would drive the respective points farther into the area of the BPT diagram that they currently inhabit.

To ascertain the significance of the results using only data from the outer bin, we repeat the statistical tests described previously. We achieved results as or more different than those observed in $<1 \%$ of the cases; in only $13 \%$ of the cases do the subsamples separate into the two different regions of the BPT diagram. Again, we expect these two tests to bracket the actual statistical significance. Although the statistical significance using this smaller sample is somewhat lower than that found for the entire sample, the results are entirely consistent and are now independent of any data at $r_{p}<22 \mathrm{kpc}$. We conclude that the measurements for the full sample $\left(10<r_{p} / \mathrm{kpc}<50\right)$ cannot solely be ascribed to contamination at small $r_{p}$, although at sufficiently small $r_{p}$ contamination must become a concern.

Another concern is that at a fixed projected radius we are probing physically different regions for galaxies with different total masses. To address this concern, we redo the analysis using a projected radius bin defined in units of scaled rather than physical radii. We set the scaled radius to be the ratio of the projected radius to the viral radius $r_{s}=r_{p} / r_{\text {vir }}$. To estimate the halo virial radius, we use a calibration obtained from the mean relation between luminosity and virial radius derived using the catalog of cosmological simulations that we used in Paper II. Namely, that catalog is based on halo merger trees from the Bolshoi-Planck simulation (Klypin et al. 2016; Rodríguez-Puebla et al. 2016), with halos found using the ROCKSTAR phase-space halo finder (Behroozi et al. 2013a) and merger trees generated with the CONSISTENT TREES code (Behroozi et al. 2013b), and finally stellar masses modeled with the UNIVERSEMACHINE code (Behroozi et al. 2018). For a Milky Way like galaxy, the $10<$ $r_{p} / \mathrm{kpc}<50$ range corresponds to $0.05<r_{s}<0.25$, and we adopt this as the range of scale radii and require $r_{p}>10 \mathrm{kpc}$, applied using the estimated virial radius of each individual galaxy. This criterion replaces the $10<r_{p} / \mathrm{kpc}<50$ criterion, so the size of the sample of sightlines will differ slightly, with mean value of $r_{p} \sim 46 \mathrm{kpc}$ in the range of $10<r_{p} / \mathrm{kpc}<136$. In Figure 3 we present the line ratios for the stacked spectra of gas within 


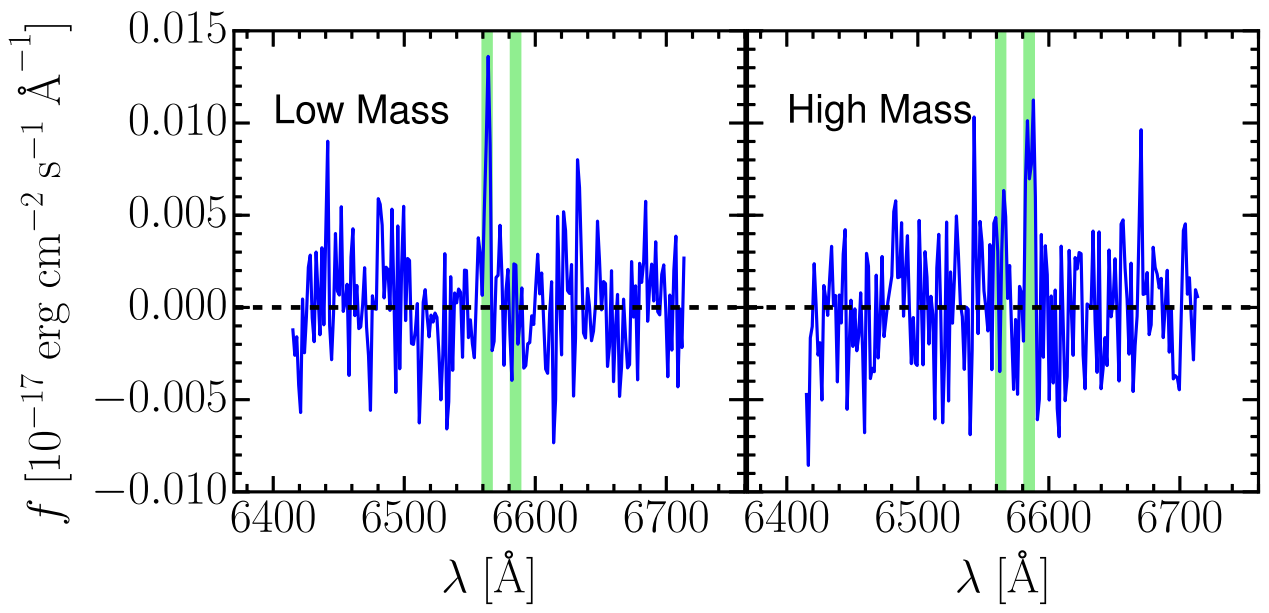

Figure 2. Stacked composite spectra for lines of sight with $10<r_{p}<50 \mathrm{kpc}$ for the spectral region that includes $\mathrm{H} \alpha$ and [N II]. The shaded regions indicate the velocity windows for those emission lines. The left panel is for the low-mass subsample and the right panel is for the high-mass subsample. The spectra appear noisier than our quantitative measurements because we reject outliers in our stacks only within the $\mathrm{H} \alpha$ and $\mathrm{N}$ [II] windows.

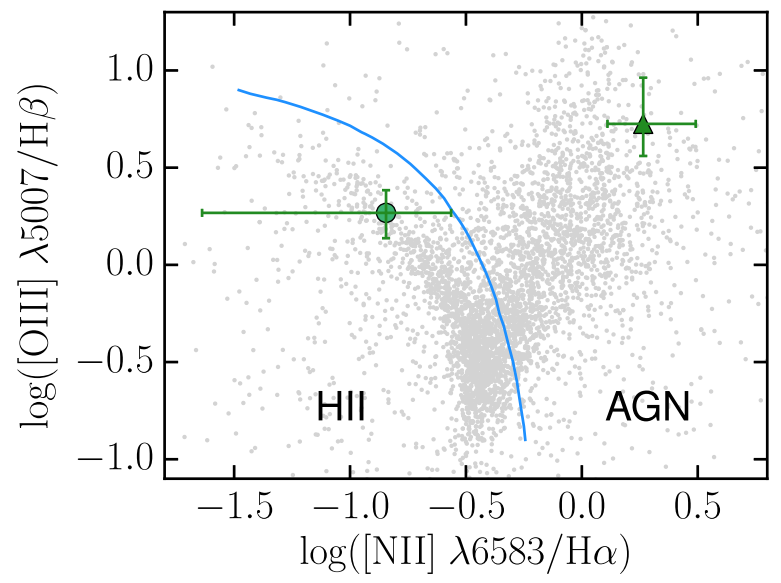

Figure 3. BPT diagram for circumgalactic gas within $0.05<r_{s}<0.25$ and $r_{p}>10 \mathrm{kpc}$. The large circle and triangle represent the measurements for galaxies with stellar mass below and above $10^{10.4} M_{\odot}$, respectively. The blue curve is the demarcation between ratios indicating ionization by star formation and AGN/shocks (Kauffmann et al. 2003a), with labels indicating which region corresponds to each of the two mechanisms. The light gray points represent the line ratios for the integrated central parts of individual galaxies as measured by SDSS. The $\mathrm{H} \beta$ flux is estimated using the $\mathrm{H} \alpha$ flux as described in the text.

$0.05<r_{s}<0.25$ and $r_{p}>10 \mathrm{kpc}$, for galaxies binned by mass above and below $M_{*}=10^{10.4} M_{\odot}$. Qualitatively, the results are unchanged, although the uncertainties in these new measurements are somewhat smaller, possibly suggesting that there is less scatter in properties when considering scaled rather than physical radii.

Using the same simulation data, we return to the issue of the velocity integration window. We replace the fixed width window with one of \pm half the virial velocity, estimated from a scaling of the galaxy luminosity. The resulting average velocity windows are $\pm 80 \mathrm{~km} \mathrm{~s}^{-1}$ and $\pm 145 \mathrm{~km} \mathrm{~s}^{-1}$ for the low- and high-mass subsamples, respectively. We find no change in the results that affects our conclusions, although the resulting measurement uncertainties are larger.

Galaxy properties are interconnected, and so it is difficult to unambiguously isolate a single driver for any observed behavior. We favor mass here because of the theoretical work, but other factors that track mass, such as star formation, could be playing a role. To test for this, we set out to measure any residual correlation with SFR separately within the low- and high-mass samples. We find no significant differences between equally populated low $\left(-4<\log \left(\mathrm{SFR} /\left(M_{\odot} / \mathrm{yr}\right)\right)<1\right)$ and high $\left(1<\log \left(\mathrm{SFR} /\left(M_{\odot} / \mathrm{yr}\right)\right)<3\right)$ SFR subsamples populated groups within each of our two mass bins. In both mass bins, the high and low SFR samples remain consistent with the results from the full samples.

\section{Conclusions}

We present measurements of diagnostic line ratios of the line emitting warm gas in the CGM of normal, nearby galaxies. Those line ratios indicate that lower-mass galaxies, $M_{*}<$ $10^{10.4} M_{\odot}$, have halo gas that is ionized by softer sources, similar to those found in star-forming regions, while highermass galaxies, $M_{*}>10^{10.4} M_{\odot}$, have halo gas that is ionized by harder sources, similar to found in AGN-hosting galaxies or in shocked regions. This is yet another way in which low- and high-stellar mass galaxies distinguish themselves from each other.

Much of the theoretical work aiming to explain the general division of galaxy characteristics at a threshold stellar mass of $\sim 10^{10.4} M_{\odot}$ has focused on the nature of gas accretion onto galaxies. The expectation from that work is that lowermass galaxies have a less interrupted flow of gas to the central galaxy, leading to continual star formation, while higher-mass galaxies have inflowing gas that tends to shock and heat at large radius, interrupting the fuel flow to the central galaxy. Our observational results support this scenario, but we note the expected complexity of any full model of the baryon cycle.

Our result is based on stacking thousands of lines of sight around thousands of nearby galaxies, and as such provides no details on the range of behavior from one galaxy to the next. However, we have empirically demonstrated that the nature of the circumgalactic gas can be explored in this manner and inform what would be required to do so on a case-by-case basis. Analogous investigations will be possible for individual galaxies with the next generation of ground-based large telescopes. We will be able to examine how these line ratios vary around galaxies and how they vary from galaxy to galaxy as a function of environment. Empirically tracing the nature of fuel flowing onto the central, luminous portions of galaxies will establish, refute, or necessitate revisions of what we envision is occurring at these critical scales. 
D.Z. and H.Z. acknowledge financial support from NSF grant AST-1311326. J.W. acknowledges support from a 2018 Alfred P. Sloan Research Fellowship. The authors gratefully acknowledge the SDSS-III team for providing a valuable resource to the community. Funding for SDSS-III has been provided by the Alfred P. Sloan Foundation, the Participating Institutions, the National Science Foundation, and the U.S. Department of Energy Office of Science. The SDSS-III web site is http://www.sdss3.org/.

SDSS-III is managed by the Astrophysical Research Consortium for the Participating Institutions of the SDSS-III Collaboration including the University of Arizona, the Brazilian Participation Group, Brookhaven National Laboratory, Carnegie Mellon University, University of Florida, the French Participation Group, the German Participation Group, Harvard University, the Instituto de Astrofisica de Canarias, the Michigan State/Notre Dame/JINA Participation Group, Johns Hopkins University, Lawrence Berkeley National Laboratory, Max Planck Institute for Astrophysics, Max Planck Institute for Extraterrestrial Physics, New Mexico State University, New York University, Ohio State University, Pennsylvania State University, University of Portsmouth, Princeton University, the Spanish Participation Group, University of Tokyo, University of Utah, Vanderbilt University, University of Virginia, University of Washington, and Yale University.

\section{ORCID iDs}

Huanian Zhang (张华年) (1D https://orcid.org/0000-00020123-9246

Dennis Zaritsky (D) https://orcid.org/0000-0002-5177-727X

Jessica Werk (i) https://orcid.org/0000-0002-0355-0134

Peter Behroozi (1D https://orcid.org/0000-0002-2517-6446

\section{References}

Alam, S., Albareti, F. D., Allende Prieto, C., et al. 2015, ApJS, 219, 12 Anglés-Alcázar, D., Faucher-Giguère, C.-A., Kereš, D., et al. 2017, MNRAS, 470, 4698

Baker, J. G., \& Menzel, D. H. 1938, ApJ, 88, 52

Baldwin, J. A., Phillips, M. M., \& Terlevich, R. 1981, PASP, 93, 5

Behroozi, P., Wechsler, R., Hearin, A., \& Conroy, C. 2018, arXiv:1806.07893
Behroozi, P. S., Wechsler, R. H., \& Wu, H.-Y. 2013a, ApJ, 762, 109

Behroozi, P. S., Wechsler, R. H., Wu, H.-Y., et al. 2013b, ApJ, 763, 18

Birnboim, Y., \& Dekel, A. 2003, MNRAS, 345, 349

Blanton, M. R., Hogg, D. W., Bahcall, N. A., et al. 2003, ApJ, 594, 186

Bordoloi, R., et al. 2011, ApJ, 743, 10

Brinchmann, J., Charlot, S., White, S. D. M., et al. 2004, MNRAS, 351, 1151

Croft, R. A. C., Miralda-Escudé, J., Zheng, Z., et al. 2016, MNRAS, 457, 3541

Croft, R. A. C., Miralda-Escudé, J., Zheng, Z., Blomqvist, M., \& Pieri, M. 2018, MNRAS, 481, 1320

Dekel, A., \& Birnboim, Y. 2006, MNRAS, 368, 2

Ford, A. B., Davé, R., Oppenheimer, B. D., et al. 2014, MNRAS, 444, 1260

Gallazzi, A., Charlot, S., Brinchmann, J., White, S. D. M., \& Tremonti, C. A. 2005, MNRAS, 362, 41

Hummer, D. G., \& Storey, P. J. 1987, MNRAS, 224, 801

Joshi, R., Srianand, R., Petitjean, P., \& Noterdaeme, P. 2018, MNRAS, 476,210

Kauffmann, G., Heckman, T. M., Tremonti, C., et al. 2003a, MNRAS, 346, 1055

Kauffmann, G., Heckman, T. M., White, S. D. M., et al. 2003b, MNRAS, 341, 54

Kereš, D., Katz, N., Weinberg, D. H., \& Davé, R. 2005, MNRAS, 363, 2

Kewley, L. J., Dopita, M. A., Sutherland, R. S., Heisler, C. A., \& Trevena, J. 2001, ApJ, 556, 121

Klypin, A., Yepes, G., Gottlöber, S., Prada, F., \& Heß, S. 2016, MNRAS, 457, 4340

Lan, T.-W., \& Mo, H. 2018, arXiv:1806.05786

Ménard, B., Scranton, R., Fukugita, M., \& Richards, G. 2010, MNRAS, 405, 1025

Ménard, B., Wild, V., Nestor, D., et al. 2011, MNRAS, 417, 801

Oppenheimer, B. D., Segers, M., Schaye, J., Richings, A. J., \& Crain, R. A. 2018, MNRAS, 474, 4740

Osterbrock, D. E., \& Ferland, G. J. 2006, Astrophysics of Gaseous Nebulae and Active Galactic Nuclei

Prochaska, J. X., Werk, J. K., Worseck, G., et al. 2017, ApJ, 837, 169

Roberts, M. S., \& Haynes, M. P. 1994, ARA\&A, 32, 115

Rodríguez-Puebla, A., Behroozi, P., Primack, J., et al. 2016, MNRAS, 462,893

Steidel, C. C., Erb, D. K., Shapley, A. E., et al. 2010, ApJ, 717, 289

Tumlinson, J., Peeples, M. S., \& Werk, J. K. 2017, ARA\&A, 55, 389

Veilleux, S., \& Osterbrock, D. E. 1987, ApJS, 63, 295

Werk, J. K., Prochaska, J. X., Cantalupo, S., et al. 2016, ApJ, 833, 54

Werk, J. K., Prochaska, J. X., Tumlinson, J., et al. 2014, ApJ, 792, 8

White, S. D. M., \& Rees, M. J. 1978, MNRAS, 183, 341

Zaritsky, D. 1994, AJ, 108, 1619

Zhang, H., Zaritsky, D., \& Behroozi, P. 2018, ApJ, 861, 34

Zhang, H., Zaritsky, D., Zhu, G., Ménard, B., \& Hogg, D. W. 2016, ApJ, 833,276

Zhu, G., \& Ménard, B. 2013a, ApJ, 773, 16

Zhu, G., \& Ménard, B. 2013b, ApJ, 770, 130 